Relations industrielles

Industrial Relations

\title{
Manuels d'histoire et syndicalisme au Québec
}

\section{James Thwaites}

Volume 35, numéro 2, 1980

URI : https://id.erudit.org/iderudit/029067ar

DOI : https://doi.org/10.7202/029067ar

Aller au sommaire du numéro

\section{Éditeur(s)}

Département des relations industrielles de l'Université Laval

\section{ISSN}

0034-379X (imprimé)

1703-8138 (numérique)

Découvrir la revue

\section{Citer cet article}

Thwaites, J. (1980). Manuels d'histoire et syndicalisme au Québec. Relations industrielles / Industrial Relations, 35(2), 321-327.

https://doi.org/10.7202/029067ar

Tous droits réservés @ Département des relations industrielles de l'Université Laval, 1980
Ce document est protégé par la loi sur le droit d'auteur. L'utilisation des services d'Érudit (y compris la reproduction) est assujettie à sa politique d'utilisation que vous pouvez consulter en ligne.

https://apropos.erudit.org/fr/usagers/politique-dutilisation/ 


\title{
Manuels d'histoire et syndicalisme au Québec
}

\author{
James Thwaites
}

\section{LES MANUELS GÉNERAUX}

Quand Marcel Trudel et Geneviève Jain ont élaboré leur étude sur les manuels d'histoire utilisés dans les écoles francophones et anglophones au Canada, au milieu des années 1960, ils ont provoqué une réflexion profonde chez leurs collègues ${ }^{1}$. Leur analyse des manuels à tous les niveaux pré-universitaires révélait une situation déplorable qu'il fallait à tout prix essayer de changer. Il ne fallait pas se surprendre, par conséquent, de trouver le nom de Marcel Trudel associé à ceux de P.G. Cornell, Jean Hamelin et Fernand Ouellet dans un projet de manuel peu de temps après. Leurs études ont donné lieu à un ouvrage innovateur, fouillé et équilibré, ainsi que disponible dans les deux langues officielles ${ }^{2}$. C'est sans doute l'esprit de ce travail qui a inspiré d'autres à tenter la même expérience au plan provincial avec certains résultats prometteurs jusqu'à ce jour.

Si le problème des manuels généraux était normalement considéré comme un de mauvaises interprétations et d'inexactitudes, en ce qui touche le mouvement ouvrier il y avait un problème d'omission. Il ne s'agissait pas de corriger l'image du mouvement ouvrier, car il n'y en avait pas. Il fallait créer une image. Ầ cet égard, je me souviens d'un manuel utilisé à l'école secondaire vers la fin des années 1950 qui nous apprenait essentiellement deux choses sur le mouvement ouvrier: que le Canada était représenté à l'OIT et que George Brown, un des pères de la confédération, avait été assassiné par un membre du syndicat des typographes de Toronto. Quant au mouvement, son évolution, ses idées, sa contribution à la société canadienne - rien du tout! Le problème n'est pas unique au Canada, car on trouve des situations semblables ailleurs: aux États-Unis où, dans l'État du Maine par exemple, le "Bureau of Labor Education" de l'Université du Maine s'est vu obligé à diffuser des brochures et films et même d'intervenir au niveau des écoles afin de sensibiliser étudiants et professeurs à cette réalité. Au printemps 1980, un congrès international à Paris abordera, entre d'autres sujets, ce même thème. En cela, le manuel général de Cornell et collaborateurs a fait un pas dans la bonne direction.

- THWAITES, J., professeur, département des relations industrielles, Université Laval.

1 Voir résumé: Marcel TRUDEL et Geneviève JAIN, «L'histoire du Canada: enquête sur les manuels", dans P.W. Bélanger et G. Rocher, éd., École et société au Québec: éléments d'une sociologie de l'éducation, Montréal, HMH, 1970, PP. 105-121.

2 CORNELL, P.G., J. HAMELIN, F. OUELLET et M. TRUDEL, Canada: unité et diversité, Toronto et Montréal, HRW, 1971, $622 \mathrm{p}$. 


\section{LES MANUELS SPÉCIALISÉS}

Curieusement, les manuels portant sur le mouvement ouvrier ont fait jusqu'à maintenant une évolution en sens inverse. Le plus récent est pire que ce que nous avions ou, au moins, il n'est certainement pas supérieur. Avant les années 1960, il existait quelques rares manuels dont ceux de Jean-Pierre Després et H.A. Logan ${ }^{3}$. Le manuel de Després était disponible uniquement en français et celui de Logan uniquement en anglais. Tous les deux se restreignaient à la période avant le milieu des années 1940. Celui de Després était synthétique, mais trop général, et portait trop sur les problèmes d'actualité de son époque. Celui de Logan était très bien sur les structures et le fonctionnement des syndicats et des centrales, mais il laissait sérieusement à désirer sur le plan socio-économique. Généralement utiles à leur époque, ces manuels ne pouvaient rien nous apprendre sur la période depuis la fin de la Seconde Guerre mondiale à cause de leurs dates de publication: 1946 et 1948 respectivement. Les événements de grande importance depuis 1945 ainsi restent sans synthèse globale.

Charles Lipton croyait avoir la réponse à nos problèmes dans son étude: The Trade Union Mouvement of Canada: 1827-1959, publié en $1967^{4}$. (Subséquemment, Michel Van Schendel a traduit cette étude en français). Le résultat, malheureusement, était loin d'être satisfaisant. Lipton comblait l'aspect socio-économique ignoré par ses prédécesseurs, mais il ignorait à son tour les aspects structurels. Un certain nombre d'interprétations tendancieuses et d'inexactitudes diminuaient la qualité de l'ouvrage. Le tout était couronné par une densité d'information et certaines lacunes de style qui rendaient difficiles la lecture et la compréhension globale du texte.

Il est possible que cette étude de Lipton ait influencé les auteurs du manuel publié par la CEQ et CSN. Certaines de ses lacunes se retrouvent ainsi dans 150 ans de luttes $^{5}$. L'information relativement limitée dans Lipton sur la situation québécoise aurait pu convaincre des chercheurs à vouloir combler ce vide. De toute façon, un manuel compréhensif et équilibré sur le plan pan-canadien est toujours à écrire.

Ce manque d'un bon manuel de base sur le mouvement ouvrier sera sans doute comblé d'ici quelques années. L'étude considérable de Eugène Forsey, entreprise il y a environ quinze ans pendant son séjour au CTC, nous éclairera sans doute sur le $19 \mathrm{e}$ siècle ${ }^{6}$. D'autres études de la sorte sont en voie d'élaboration actuellement.

3 DESPRÉS, J.-P., Le mouvement ouvrier canadien, Montréal, Fides, 1946, 205 p. H.A. LOGAN, Trades Unions in Canada: Their Functioning and Development, Toronto, Macmillan, 1948, 639 p.

4 LIPTON, C., The Trade Union Movement of Canada: 1827-1959, Toronto, N.C. Press, 1973, 384 p.

5 CEQ-CSN, 150 ans de luttes: histoire du mouvement ouvrier au Québec (1825-1976), Québec, CEQ-CSN, (1979), 235 p.

6 Cette étude est actuellement sous presses à l'Université de Toronto au moment de la rédaction de ce commentaire. 
Les historiens, en effet, ont été très actifs au cours des années 1960 et 1970 dans l'élaboration et la publication de recherches fortement pertinentes. Plusieurs monographies ont paru sous forme de volumes et de collections d'articles tels que:

I.M. ABELLA, Nationalism, Communism and Canadian Labour, Toronto, UTP, 1973, $256 \mathrm{p}$.

N. BÉLANGER et collab., Les travailleurs québécois: 1851-1896, Montréal, PUQ, 1973, $221 \mathrm{p}$.

D. BERCUSON, Confrontation at Winnipeg, Montréal and London, McGill-Queen's, 1974, 227 p.

, Fools and Wise Men: The Rise and Fall of the "One Big Union", Toronto, MH-R, 1978, $300 \mathrm{p}$.

J. DE BONVILLE, Jean-Baptiste Gagnepetit: les travailleurs montréalais à la fin du 19e siècle, Montréal, L'Aurore, 1975, $253 \mathrm{p}$.

G.S. KEALEY et P. WARRIAN, éd., Essays in Canadian Working Class History, Toronto, MS, 1976, $231 \mathrm{p}$.

A.R. MCCORMACK, Reformers, Rebels and Revolutionaries: the Western Canadian Radical Mouvement 1899-1919, Toronto, UTP, 1977, $228 \mathrm{p}$.

J. ROUILLARD, Les syndicats nationaux au Québec de 1900 à 1930, Québec, PUL, 1979, 342 p.

Cet intérêt marqué pour le syndicalisme et, plus généralement, les travailleurs et la classe ouvrière, a donné naissance à deux associations: "The Committee on Canadian Labour History" (C.C.L.H.), fondé en 1971 et le "Regroupement de chercheurs en histoire des travailleurs québécois» (R.C.H.T.Q.), fondé en 1972; ainsi que trois revues scientifiques: Bulletin of the C.C.L.H. (1975-79), Le Bulletin R.C.H.T.Q., $(1974+)$, et Labour/Le travailleur $(1976+)$. Ces associations voulaient promouvoir la recherche et fournir un forum où les chercheurs intéressés pourraient se rencontrer, échanger de l'information et établir des bases de collaboration. La production impressionnante des dernières années n'est pas nécessairement reliée directement à ces associations. Cependant, la presque totalité des chercheurs dans ce domaine se retrouvent dans l'un ou l'autre, souvent dans tous les deux. Le R.C.H.T.Q. d'ailleurs publie sa propre collection aux Presses de l'Université du Québec depuis 1973. Sept titres ont paru à date et d'autres sont sous presses.

\section{LE MANUEL DE LA CEQ ET CSN}

Le manuel de la CEQ et CSN, 150 ans de luttes: histoire du mouvement ouvrier au Québec (1825-1976), est né d'un besoin réel chez les syndiqués: vouloir comprendre l'évolution de leurs mouvements; vouloir comprendre leur contribution au pays; et vouloir les faire savoir auprès des militants aussi bien que du grand public.

Il faut noter qu'il n'existait pas de manuel au Québec qui pouvait satisfaire aux deux critères essentiels: se consacrer essentiellement à la situation québécoise, et couvrir toutes les périodes, y compris la plus récente, depuis la Seconde Guerre mondiale. 
L'objectif principal de cette étude semble avoir été de bâtir un manuel pratique afin "d'assurer la formation des militants syndicaux», (p. 7) et de contribuer ainsi «au développement de la conscience historique des travailleurs $)^{7}$. Cet objectif se comprend facilement chez un mouvement qui veut promouvoir une certaine unité de pensée et d'action. Il se comprend encore davantage si l'on tient compte de la sorte de "guerre froide» qui existait entre l'histoire en tant que discipline et le système scolaire au cours de la période postérieure à la réforme de l'éducation. On avait marginalisé, sinon éliminé, l'histoire à toute fin pratique des études pré-universitaires, comme si on voulait absolument prouver que Lord Durham avait raison après tout! La devise de la province est changée de «Je me souviens» à «Je préfère oublier».

La nouvelle orientation du Québec avait été notée et analysée entre d'autres par Michel Brunet ${ }^{8}$. Le Québec des années 1960 devait se servir de tous les moyens pour bâtir une société capable de se défendre et de se développer, en utilisant les outils industriels, technologiques, etc., disponibles, plutôt que de les rejeter - et en les mettant entre les mains des Québécois autant que possible. L'État devait jouer un rôle positif dans ce processus en tant que planificateur.

Il était peut-être normal que les gouvernants et la population québécoise, inspirés par cette nouvelle orientation, rejettent une discipline axée sur le passé pour chercher d'autres voies. D'abord, il y avait la situation des manuels, signalée par Trudel et Jain, qui laissait croire qu'on enseignait une combinaison de faits, de foutaise et de propagande. Deuxièmement, il y avait la vague de popularité de l'école de Lionel Groulx avant 1960 qu'on avait confondue avec la pensée de l'Union Nationale sous Maurice Duplessis (surtout son traditionnalisme et nationalisme), et qui semblait par conséquent incompatible avec le Québec de la Révolution tranquille. Les années 1960 étaient témoin d'une remise en question du régime précédant et de tout ce qui lui était associé 9 . Deux thèmes entr'autres ont subi une réévaluation intégrale: la société traditionnelle et le nationalisme. Et l'histoire, au niveau des manuels, était fortement imprégnée des deux.

Subséquemment, l'histoire est presque disparue des écoles à tous les niveaux pré-universitaires, et par conséquent de l'esprit de la collectivité. Même là où elle avait survécu, souvent elle était bâtardisée, étant basée de plus en plus sur l'actualité et étant de plus en plus engagée. (Pendant cette période, un de mes collègues à l'Université Vermont me disait: "Le drame des historiens au Québec est qu'ils ne peuvent décider s'ils ont l'intention d'écrire ou de faire de l'histoire.» Il n'était peut-être pas loin de la réalité pour ce qui en restait d'histoire dans les écoles).

7 SAINT-PIERRE, Céline, «Pour développer la conscience historique des travailleurs», Communiqué CEQ-CSN, Montréal et Québec, CSN-CEQ, (1979), p. 3.

8 BRUNET, M., La présence anglaise et les canadiens: études sur l'histoire et la pensée des deux Canada, Montréal, Beauchemin, 1958, 293 p.

9 Voir à titre d'exemple, la lutte menée par la revue Cité libre pendant toute son existence. 
Il y a trois ou quatre ans, le présent gouvernement a posé un geste en rendant obligatoire l'histoire nationale, après plusieurs années d'oubli. (Malheureusement, il y a beaucoup plus que l'histoire nationale et nous sommes un des rares peuples au monde aujourd'hui à ne l'avoir pas compris). Ce premier geste prometteur cependant était couronné d'une confusion regrettable: la renaissance du lien Groulx-Duplessis. On a créé un concours Lionel Groulx dont le premier sujet privilégié était Maurice Duplessis ${ }^{10}$. À peu près en même temps, on a dévoilé le monument de Maurice Duplessis, pourtant si longtemps oublié, sur la colline parlementaire. (Groulx a dû tourner dans son tombeau). Nous espérons que ces gestes ont plus qu'une raison d'être politique, mais leur orientation actuelle est sans équivoque. Duplessis n'était pas exactement l'ami de l'ouvrier.

Voilà la situation! Les syndiqués, qui étaient aux études avant le milieu des années 1960, avaient subi l'ancien régime de manuels inadéquats sans mention de la question syndicale à toute fin pratique. Ceux qui étaient aux études après cette période avaient subi le régime de "guerre froide», une véritable absence d'enseignement historique, ou au plus une histoire très engagée et très actuelle sans manuel pertinent. Tragiquement, cette période de "guerre froide» au Québec est arrivée en même temps que la formulation de manuels généraux bien conçus et d'études monographiques solides portant sur le mouvement ouvrier.

Le manuel de la CEQ et CSN est issu de cette situation. Cet effort était peut-être encouragé par la production "parallèle» des historiens sérieux aussi bien que par l'absence d'une synthèse valable et d'actualité sur l'expérience syndicale québécoise. De toute façon, le mouvement syndical avait décidé de prendre en main la publication d'un manuel sur son passé. Quant au résultat, il ne s'agit pas d'un simple travail de type «relations publiques», tel que produit trop souvent par l'entreprise privée, car l'équipe de recherche et rédaction comprend des personnes de l'intérieur et de l'extérieur des centrales. Elle semble avoir sollicité aussi la collaboration de spécialistes extérieurs pour fins de critique (p. 8). Cela veut dire que les centrales ne pouvaient se garantir le contrôle de l'information communiquée. D'ailleurs, malgré les critiques de certaines prises de position syndicales dans cet ouvrage, les présidents N. Rodrigue et R. Gaulin affichent publiquement leur enthousiasme quant au travail accompli ${ }^{11}$.

L'équipe fait également son autocritique d'une façon réaliste dans la préface et le communiqué de presse de Céline Saint-Pierre, quoique les commentaires de cette dernière auraient dû se trouver dans le texte même. Ầ titre d'exemple, Céline Saint-Pierre écrit:

10 Le concours secondaire avait comme thème: «Maurice Duplessis: le chef?» et le Concours collégial avait comme thème: «Maurice Duplessis: reflet d'un Québec traditionnel ou précurseur d'un Québec nouveau?».

11 RODRIGUE, Norbert, «Pour mieux continuer le combat aujourd'hui en tirant les leçons des combats d'hier», et Robert GAULIN, "Ce livre raconte à lui seul l'histoire de plus de monde que tous les autres manuels d'histoire résumés», Communiqué CEQ-CSN, Montréal et Québec, CSN-CEQ, 1979, 6 p. 
«Le comité est bien conscient des limites qu'il (le livre) comporte et de ses lacunes. Bien des questions telles la condition ouvrière, l'évaluation des gains obtenus par les travailleurs, les conditions de travail des femmes ont été peu développées. D'autres ont été peu abordées telles les problèmes organisationnels et l'évolution des structures syndicales et il faut souhaiter qu'elles le soient ultérieurement. Malgré l'intention du Comité de traiter de façon analytique des rapports entre la conjoncture économicopolitique et la conjoncture syndicale, il n'a pas été possible de réaliser cet objectif dans les délais impartis» ${ }^{12}$.

Un des problèmes signalés par Céline Saint-Pierre était les «immenses lacunes quant aux connaissances acquises sur l'histoire du mouvement ouvrier $»^{13}$, particulièrement grave dans un ouvrage basé sur les études existantes et non pas sur la recherche fondamentale. Elle aurait pu signaler également la valeur inégale des études publiées à ce jour. Cependant, certaines lacunes auraient pu être comblées en se référant à des études et des thèses facilement accessibles qui ne semblent pas avoir été consultées. Voici quelques exemples: la thèse de Clare Pentland sur les origines du système capitaliste au Canada (ou le résumé de cette thèse qui a paru dans Aranka Kovacs, éd., Readings in Canadian Labour Economics ${ }^{14}$ ), la thèse de John Battye sur le mouvement de neuf heures ${ }^{15}$, celle de Michel Têtu sur la C.T.C.C. ${ }^{16}$, et l'étude de Ross McCormack sur le I.W.W. ${ }^{17}$. La bibliographie brève à la fin de l'ouvrage est à la fois trop sommaire et de valeur inégale; elle mériterait d'être plus étoffée afin de fournir une idée de l'envergure de la recherche entreprise et de permettre aux lecteurs «d'aller plus loin» s'ils le désirent.

Certains sujets souffrent d'un manque d'élaboration, par exemple, l'explication du rôle du chef syndical Alfred Charpentier (p. 96) et de la centrale C.C.T. (p. 91). Certains thèmes n'ont pas été exploités suffisamment, à part ceux mentionnés par Céline Saint-Pierre, notamment la dimension légale des questions syndicales et ouvrières (pp. 96-97). Il y a dans le texte certaines fautes d'omission telles que l'explication des conditions nécessaires au développement du capitalisme, (p. 11) et les éléments de la politique nationale de Macdonald (p. 20). Il y en a d'autres d'ordre analytique, telles

12 SAINT-PIERRE, Céline, op. cit., p. 2.

13 Ibid., pp. 2-3.

14 PENTLAND, H.C., "The Development of a Capitalistic Labour Market in Canada", dans A. Kovacs, éd., Readings in Canadian Labour Economists, Toronto, M.H., 1961, pp. 2-14. H.C. PENTLAND, Labour and the Development of Industrial Capitalism, thèse de doctorat (économique), Toronto, University of Toronto, 1960, $437 \mathrm{p}$.

15 BATTYE, J., The "Nine Hour Movement" of 1872: Genesis of the Canadian Labour Movement, thèse de maîtrise (histoire), Waterloo, Waterloo University, 1971; voir également l'article récemment publié par J. BATTYE à ce sujet, "The Nine Hour Pioneers: The Genesis of the Canadian Labour Movement”, Labour/Le travailleur, vol. 4, 1979, pp. 25-26.

16 TÊTU, M., Les premiers syndicats catholiques canadiens, 1900-1921, thèse de doctorat (lettres), Québec, Université Laval, 1961, 562 p.

17 MC CORMACK, A.R., Reformers, Rebels and Revolutionaries: The Western Canadian Radical Movement 1899-1919, Toronto, UTP, 1977, 228 p. 
que l'interprétation de la portée de l'argumentation de Normand Séguin (p. 22), celle du rôle de la F.A.T. dans la défaite des Chevaliers du travail (p. 43) et celle du degré de radicalisme chez les travailleurs pendant la dépression des années 1930 (p. 86).

Il serait souhaitable dans les versions futures de l'ouvrage d'éliminer les éléments de confusion. À titre d'exemple, les photos de la page couverture et de la page 77 devraient faire place aux photos appropriées sur les manifestations syndicales (il s'agit d'une photo d'une manifestation en faveur de la guerre Sud-africaine!). Une autre photo laisse à désirer à la page 115. Mgr. Charbonneau n'est sûrement pas «typique» du conservatisme de l'Église! Voici encore des inexactitudes:

- Borden n'était pas à la tête des «bleus» mais des «unionistes» pendant la crise de la conscription (p. 56)

- Bourassa était lié aux idées de nationalisme pan-canadien longtemps avant 1921 (voir le programme de la Ligue nationaliste canadienne 1903) (p. 57)

- le réseau de chemins de fer Canadien national n'existait pas en 1910; il s'agit du Grand Tronc (p. 64)

- on s'est servi des échelles salariales de 1926-29 en 1941 car ces échelles étaient supérieures à tous ce qui existait de 1929 à 1940 à cause de la dépression (p. 103).

Afin d'améliorer ce manuel pour les éditions futures, il faudrait effectuer également quelques changements d'ordre général. La structure du texte devrait être revue afin de ne pas défavoriser des périodes du passé de grande importance et afin d'atténuer l'accent indu sur les périodes plus récentes tout en traitant le tout avec plus de rigueur scientifique. Il faudrait faire attention à la sélection des événements clefs et uniformiser l'argumentation générale présentée dans le texte, tout en diminuant la tendance apologétique de l'ouvrage. Ce manuel devrait inclure quelques tableaux, des notes de références explicatives là où elles sont pertinentes, et une conclusion mieux adaptée au texte.

$\mathrm{Au}$ dire de l'équipe de recherche et de rédaction, le projet entrepris n'est pas terminé et se continuera. On aura alors l'occasion d'améliorer ce manuel que l'on veut apte à jouer le rôle que l'on se propose auprès des militants et du grand public. Â cet égard, je suis disposé à fournir aux auteurs des commentaires détaillés sur tous les points signalés et d'autres s'ils sont intéressés.

Une question avant de terminer. Il serait utile de connaître les raisons derrière le refus de la F.T.Q. de participer au projet initial et la décision de celle-ci de lancer son propre projet sous la direction de Léo Roback. S'agitil d'un problème relié au rôle de la Fédération comparé à celui (ou ceux) des centrales? S'agit-il de différences d'interprétation? Ou s'agit-il de divergences idéologiques? Afin de répondre à ces questions et de mieux comprendre le manuel de la CEQ et CSN, on attend avec beaucoup d'intérêt la publication parrainée par la FTQ. 